\title{
Empowering geoscientists to improve diversity and inclusion in the geosciences: The case of ADVANCEGeo Partnership to promote Institutional and cultural change
}

\author{
ASMERET ASEFAw BERHE
}

\begin{abstract}
Geosciences remains one of the least diverse fields, even within the science, technology, engineering and mathematics (STEM). Demographics of the geosciences workforce and student body in the U.S. continue to show severe underrepresentation of women, African-Americans, HispanicAmericans, Native-Americans/Native-Alaskans and persons with disabilities in particularly in the geosciences. Among the documented causes of the persistent lack of diversity in the geosciences include bias, discrimination, and harassment of members of underrepresented groups. These behaviors persist due to power imbalances, continued marginalization of underrepresented groups, and inadequate policies against misconduct in academic and other scientific institutions. Academic institutions and professional societies can transform scientific culture through codifying professional practice, recognizing contributions to the discipline, providing opportunities for mentorship and networking, and advocating for policy change. Here we discuss recent initiatives by scientific societies and funding agencies in the U.S., including our work with ADVANCEGeo, to improve diversity, equity and inclusion through efforts aimed at increasing retention and advancement of diverse communities of scientists. We draw on research funded in part by the ADVANCE program to discuss successful strategies and highlight the role of collaborations among societies for creating cultural and institutional change at the national and international levels.
\end{abstract}

